\title{
Modelling the effects of calcium waves and oscillations on saliva secretion
}

\author{
Laurence Palk $^{\mathrm{a}, *}$, James Sneyd ${ }^{\mathrm{a}}$, Kate Patterson ${ }^{\mathrm{a}}$, Trevor J. Shuttleworth ${ }^{\mathrm{b}}$, David I. Yule ${ }^{\mathrm{b}}$, \\ Oliver Maclaren ${ }^{\mathrm{c}}$, Edmund J. Crampin ${ }^{\mathrm{c}}$ \\ a Department of Mathematics, The University of Auckland, Private Bag 92019, Auckland 1142, New Zealand \\ b Department of Pharmacology and Physiology and the Centre for Oral Biology, University of Rochester Medical Center, Rochester, NY 14642, USA \\ c Auckland Bioengineering Institute and Department of Engineering Science, The University of Auckland, Private Bag 92019, Auckland, New Zealand
}

\section{A R T I C L E I N F O}

\section{Article history:}

Received 4 November 2011

Received in revised form

5 April 2012

Accepted 6 April 2012

Available online 14 April 2012

Keywords:

Mathematical model

Parotid acinar cell

Oscillation frequency

$\mathrm{Ca}^{2+}$ wave speed

Calcium signalling

\begin{abstract}
A B S T R A C T
An understanding of $\mathrm{Ca}^{2+}$ signalling in saliva-secreting acinar cells is important, as $\mathrm{Ca}^{2+}$ is the second messenger linking stimulation of cells to production of saliva. $\mathrm{Ca}^{2+}$ signals affect secretion via the ion channels located both apically and basolaterally in the cell. By approximating $\mathrm{Ca}^{2+}$ waves with periodic functions on the apical and basolateral membranes, we isolate individual wave properties and investigate them for their effect on fluid secretion in a mathematical model of the acinar cell. Mean $\mathrm{Ca}^{2+}$ concentration is found to be the most significant property in signalling secretion. Wave speed was found to encode a range of secretion rates. $\mathrm{Ca}^{2+}$ oscillation frequency and amplitude had little effect on fluid secretion.
\end{abstract}

(c) 2012 Elsevier Ltd. All rights reserved.

\section{Introduction}

A problem commonly encountered in quantitative analysis of physiological processes is to determine which experimentally observed behaviours are important to the system and which can be approximated to produce a simple model capable of making predictions and increasing understanding. The salivation process is initiated with an electrical signal from the brain which releases an agonist, ACh, around the acinar cells. This agonist causes production of $\mathrm{IP}_{3}$ which releases $\mathrm{Ca}^{2+}$ from internal stores in the endoplasmic reticulum (ER). $\mathrm{Ca}^{2+}$ feedback on $\mathrm{IP}_{3}$ dynamics can cause periodic oscillations of $\mathrm{Ca}^{2+}$ throughout the cytoplasm at a raised baseline. The raised $\mathrm{Ca}^{2+}$ concentration opens $\mathrm{K}^{+}$and $\mathrm{Cl}^{-}$channels, causing a change in intracellular and lumenal concentrations of $\mathrm{Cl}^{-}, \mathrm{Na}^{+}$and $\mathrm{K}^{+}$. This concentration change creates an osmotic gradient which leads to increasing fluid secretion from the acinar cells. This process is completed in a great many acinar cells simultaneously and is accompanied by a shrinking of cell volume. Once the fluid is secreted from the acinar cells into the lumen as primary saliva it travels down the parotid ducts where duct cells modify the ionic content before finally being secreted in the mouth.

One mechanism that is particularly well studied is that of the $\mathrm{Ca}^{2+}$ dynamics. $\mathrm{Ca}^{2+}$ is well known to have an important role as

\footnotetext{
${ }^{*}$ Corresponding author. Tel.: +6499232448.

E-mail address: 1.palk@math.auckland.ac.nz (L. Palk).
}

a second messenger in a vast array of cell types. Current models of saliva secretion in the parotid acinar cells by Palk et al. (2010) and Gin et al. (2007) use compartmental models of $\mathrm{Ca}^{2+}$ to reproduce experimental results and incorporate into models for saliva secretion. These models assume homogeneous oscillations in $\mathrm{Ca}^{2+}$ throughout the cytosol and hence can be modelled using ordinary differential equations. Experimentally, however, $\mathrm{Ca}^{2+}$ is not only observed to oscillate but to travel in waves from one membrane to the other. These $\mathrm{Ca}^{2+}$ waves have been seen in many cells types including cardiac myocytes, airway smooth muscle, pancreatic acinar cells, neurons (Jaffe, 1991) and parotid acinar cells (Won et al., 2007). $\mathrm{Ca}^{2+}$ waves and oscillations are thought to be able to encode a larger amount of signalling information than a constant $\mathrm{Ca}^{2+}$ concentration. Experimental and theoretical evidence suggests that frequency and amplitude are used to encode information in certain cell types (De Koninck and Schulman, 1998; Tang and Othmer, 1995; Berridge, 1997). It is not our focus here to investigate the genesis of $\mathrm{Ca}^{2+}$ waves, for that see Sneyd et al. (2003). In this paper we seek to investigate how important the properties of $\mathrm{Ca}^{2+}$ waves are for controlling the secretion of primary saliva.

Consideration of $\mathrm{Ca}^{2+}$ waves, as opposed to homogeneous oscillations, requires consideration of amplitude, mean concentration, frequency and also the wave speed as mechanisms for signalling. Using a detailed spatial model of the $\mathrm{Ca}^{2+}$ waves makes it difficult to change one property, say the wave speed, without affecting the others. A spatial modelling approach involves numerically solving partial differential equations throughout the cytosol. 
Yet as regards saliva secretion, $\mathrm{Ca}^{2+}$ acts on ion channels which are located in the apical and basal membranes only. Hence using a spatial model generates far more information than is required and is not the approach taken here. Rather, we approximate $\mathrm{Ca}^{2+}$ waves by using periodic functions for $\mathrm{Ca}^{2+}$ at the basal and apical membranes. Using this approximation of $\mathrm{Ca}^{2+}$ waves we are able to isolate $\mathrm{Ca}^{2+}$ wave properties such as frequency, amplitude, wave speed and mean concentration to individually investigate their effect on saliva secretion.

\section{Modelling agonist-induced saliva secretion}

We use the mathematical model of the parotid acinar cell from Palk et al. (2010). Here saliva secretion is initiated by a raised $\mathrm{Ca}^{2+}$ concentration which open $\mathrm{K}^{+}$and $\mathrm{Cl}^{-}$channels. This enables an ionic gradient to be maintained which allows water to flow by osmosis both transcellularly and paracellularly into the lumen. A schematic of the fluid secretion model can be seen in Fig. 1.

Being un-buffered we assume that $\mathrm{K}^{+}, \mathrm{Cl}^{-}$and $\mathrm{Na}^{+}$diffuse very quickly and therefore these ionic concentrations are homogeneous throughout the three sub-domains, the interstitium, the cytosol and the lumen.

Transmembrane ion fluxes are driven by $\mathrm{Ca}^{2+}$ with the $\mathrm{Ca}^{2+}$ concentration at the apical membrane affecting the open probability of the ion channels that reside in the apical membrane and similarly the $\mathrm{Ca}^{2+}$ concentration at the basal membrane affecting the ion channels there.

Differential equations are written for the change in $\mathrm{Cl}^{-}, \mathrm{K}^{+}$, $\mathrm{Na}^{+}$, cell volume and the apical and basal membrane potentials. These are numerically solved using the Matlab routine ode15s. Foskett (1990) shows that volume changes are tightly correlated with changes in cytosolic $\mathrm{Cl}^{-}$. Our model assumes fluid flow to change instantaneously with ionic changes, and cell volume to follow directly. Details of the model equations are given in Appendix A.

\section{Simplified model of $C a^{2+}$ waves}

We consider a periodic $\mathrm{Ca}^{2+}$ wave from the apical to basal membrane with a constant period such as that seen experimentally by Zimmermann and Walz (1999). At any point throughout the cytosol the concentration of $\mathrm{Ca}^{2+}$ will be a periodic function

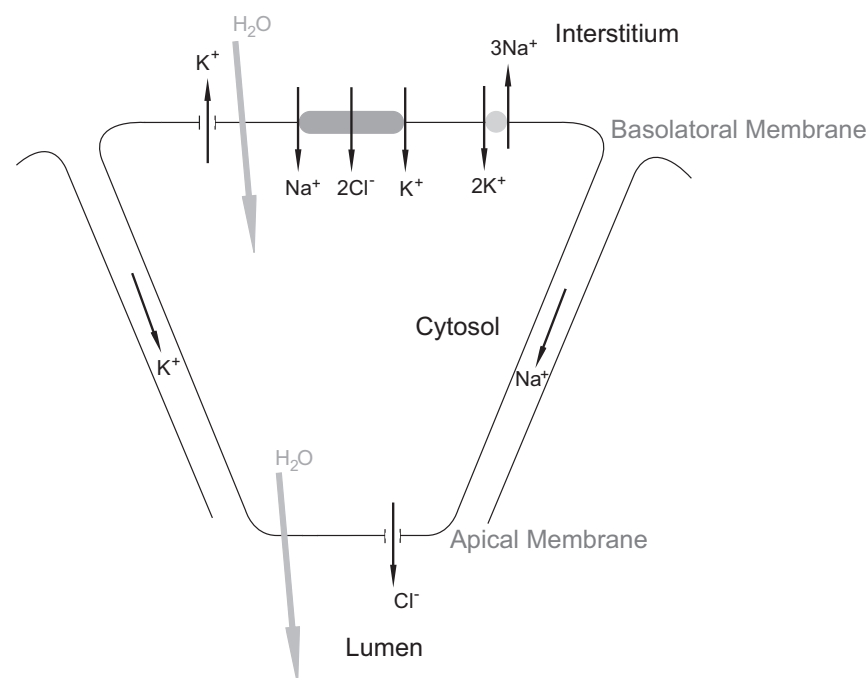

Fig. 1. A schematic of the movement of ions responsible for saliva secretion.

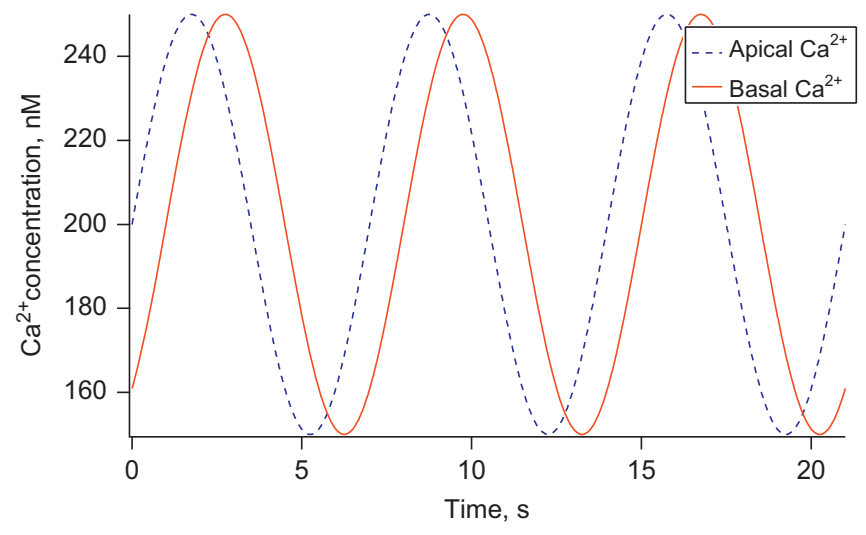

Fig. 2. $\mathrm{Ca}^{2+}$ concentrations at the apical and basal membranes numerically simulated with sine waves, period $7 \mathrm{~s}$, with mean $200 \mathrm{nM}$ and $50 \mathrm{nM}$ amplitude. There is a $1 \mathrm{~s}$ time difference between the apical and basal $\mathrm{Ca}^{2+}$ peaks which is equivalent to a $25 \mu \mathrm{m} / \mathrm{s}$ wave speed.

with the same period and a possibly distinct mean and amplitude. We simulate a $\mathrm{Ca}^{2+}$ wave with the concentration being a periodic function at both the apical and basal membranes. We can formally write this as follows:

$C_{a}=f(t)$,

$C_{b}=g(t+\delta)$,

where $C_{a}$ is the $\mathrm{Ca}^{2+}$ concentration at the apical membrane and with $C_{b}$ the basal $\mathrm{Ca}^{2+}$ concentration. Both $f(t)$ and $g(t)$ are assumed to be periodic with the same period $T$ and both attain their minimum values at $t=0$. The parameter $\delta$ is a measure of synchronicity, when $\delta=0 \mathrm{Ca}^{2+}$ oscillations are synchronous at the two membranes. When parameter $\delta$ is non-zero there is a delay between $\mathrm{Ca}^{2+}$ peaking at the apical and basal membranes. This phase-shift can be used to simulate a $\mathrm{Ca}^{2+}$ wave with a given speed. Using this model we are free to change individual wave properties, for example the wave amplitude, without affecting the other wave properties.

In Fig. 2 we simulate an apical to basal $\mathrm{Ca}^{2+}$ wave, periodic with a period of $7 \mathrm{~s}$, where the mean $\mathrm{Ca}^{2+}$ concentration and amplitude is the same at both membranes. Here a sine function was used to give the $\mathrm{Ca}^{2+}$ profile at both membranes. However, any periodic function with a profile similar to the experimentally observed $\mathrm{Ca}^{2+}$ oscillations could have been used. For the remainder of the results presented a sine function is used to approximate the oscillations of $\mathrm{Ca}^{2+}$ at the apical and basal membranes. The $\mathrm{Ca}^{2+}$ concentration $C$ is given by

$C=C_{\text {norm }}+C_{\text {amp }} \sin \left(\frac{2 \pi(t-\delta)}{\lambda}\right)$,

where $C_{\text {norm }}$ is the mean $\mathrm{Ca}^{2+}$ concentration, $C_{\mathrm{amp}}$ is the amplitude of the $\mathrm{Ca}^{2+}$ oscillations, $\delta$ allows for the inclusion of a time delay and $\lambda$ is the period of oscillations.

\section{Analysis of the effect of $\mathrm{Ca}^{2+}$ wave speed on fluid flow}

Previous work has investigated how the frequency of $\mathrm{Ca}^{2+}$ oscillations may encode signalling information in the phosphorylation of a cellular substrate by protein kinase (Goldbeter et al., 1990) and in hepatocytes (Larsen et al., 2004). We investigate the effect of wave speed on saliva secretion by varying the time difference between the peak in $\mathrm{Ca}^{2+}$ at the apical and basal membranes. Experimentally, Won et al. (2007) report a wave speed of $27.81 \mu \mathrm{m} / \mathrm{s}$ with $\mathrm{Ca}^{2+}$ peaking at the apical membrane 


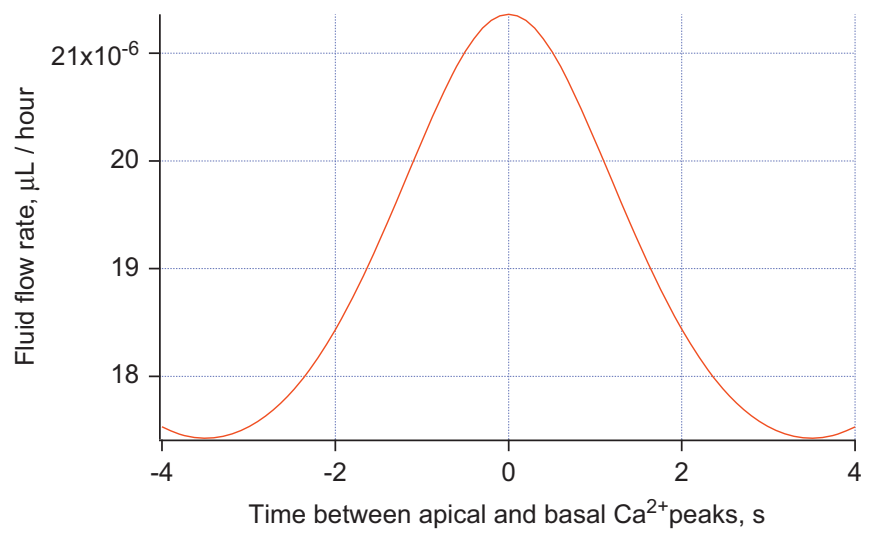

Fig. 3. Fluid flow rate against time difference between apical and basal $\mathrm{Ca}^{2+}$ peaks. Maximum secretion occurs when the apical and basal oscillations are synchronous. $\mathrm{Ca}^{2+}$ waves are approximated using a sine function with $150 \mathrm{nM}$ mean, $100 \mathrm{nM}$ amplitude and $7 \mathrm{~s}$ period.

approximately $1 \mathrm{~s}$ before the basal membrane. These measurements suggest the distance between the two membranes is $27.81 \mu \mathrm{m}$ : for simplification this work uses a distance of $25 \mu \mathrm{m}$ between membranes.

In Fig. 2 we numerically simulate an apical to basal $\mathrm{Ca}^{2+}$ wave having a $1 \mathrm{~s}$ time difference between the apical and basal $\mathrm{Ca}^{2+}$ peaks. With our assumed cell size of $25 \mu \mathrm{m}$ from the apical to basal membrane this is equivalent to a wave speed of $25 \mu \mathrm{m} / \mathrm{s}$. If instead we ran a simulation with a $2 \mathrm{~s}$ time between the apical and basal membrane peaks in $\mathrm{Ca}^{2+}$ this would approximate a wave speed of $12.5 \mu \mathrm{m} / \mathrm{s}$, assuming the same cell size. Using this idea of changing the time between apical and basal $\mathrm{Ca}^{2+}$ peaks we can simulate a range of wave speeds and observe the effect on secretion.

In Fig. 3 the effect of the time between apical and basal $\mathrm{Ca}^{2+}$ peaks can be seen on the average fluid flow rate. It is shown that maximum secretion occurs when the time difference is zero, implying synchronous $\mathrm{Ca}^{2+}$ oscillations at the two-membranes, or equivalently a homogeneous rise and fall of $\mathrm{Ca}^{2+}$ throughout the cytosol. A minimum secretion rate occurs at a time difference of $3.5 \mathrm{~s}$. This is a time difference of exactly half the oscillation period and oscillations at the two membranes are out of phase.

For this result, and the remainder of the analysis, a sinusoidal function was used to approximate the $\mathrm{Ca}^{2+}$ oscillations at each membrane. However, the same result can be reproduced for several different periodic functions at both membranes. A mathematical argument that a local maximum occurs when oscillations are synchronous for any periodic function is given in Appendix B.

The result that synchronous oscillations are most efficient appears to suggest that the experimentally observed $\mathrm{Ca}^{2+}$ waves seen by Won et al. (2007), with a 1 second time difference between apical and basal $\mathrm{Ca}^{2+}$ peaks, are less than efficient at signalling saliva secretion. There are, however, some assumptions made in the analysis above that we now explore. In particular the model currently has all ion channels operating at steady state. However, experimentally the ion channels have a time dependence. We will address this model shortfall in the following section.

\section{Time-dependent $\mathrm{Cl}^{-}$channel gating}

In Arreola et al. (1996) the $\mathrm{Cl}^{-}$channels are found to react very quickly to changes in $\mathrm{Ca}^{2+}$ at physiologically realistic membrane potentials and $\mathrm{Ca}^{2+}$ concentrations. This quick opening and closing led us to initially use a steady-state model for the $\mathrm{Cl}^{-}$ channel. We investigate whether adding the time dependence of the $\mathrm{Cl}^{-}$channel to the model affects the results relating to fluid secretion.

In Arreola et al. (1996) a four-state model is given for the $\mathrm{Cl}^{-}$ channel with three closed and one open state as follows:

$C_{1} \underset{\beta_{1}}{\stackrel{\alpha_{1} C a}{\rightleftharpoons}} C_{2} \underset{\beta_{1}}{\stackrel{\alpha_{1} C a}{\rightleftharpoons}} C 3 \underset{\beta_{2}}{\stackrel{\alpha_{2}}{\rightleftharpoons}} O$

Rates $\alpha_{1}$ and $\beta_{1}$ are faster than $\alpha_{2}$ and $\beta_{2}$ and their dependence on $\mathrm{Ca}^{2+}$ is not given explicitly in Arreola et al. (1996). Hence we simplify this model to a two-state model using a rapid equilibrium approximation to group the three closed states, $C_{1}, C_{2}$ and $C_{3}$ into one new closed state $C$.

$C \underset{\beta_{2}}{\stackrel{\alpha}{\rightleftharpoons}} O$.

This two-state model simplification approximates the experimental data well (result not shown). Applying the two-state reduction we get a differential equation for the fraction of open $\mathrm{Cl}^{-}$channels

$\frac{d O}{d t}=\alpha C-\beta_{2} O$.

Here $\beta_{2}$ is the same reverse rate as seen in Arreola et al. (1996). The forward reaction rate, $\alpha$, given in terms of the original rates $K_{1}, K_{2}$ and $\alpha_{2}$ in Arreola et al. (1996), is shown as follows:

$\alpha=\alpha_{2} /\left(1+\frac{K_{1}}{C_{a}}+\frac{K_{1}^{2}}{C_{a}^{2}}\right)$

Here

$K_{1}=214 \exp \left(\frac{-0.13 F V_{a}}{R T}\right) n M$,

$K_{2}=0.58 \exp \left(\frac{-0.24 F V_{a}}{R T}\right)$.

$\beta_{2}=K_{2} \alpha_{2} \mathrm{~s}^{-1}, \alpha_{2}=4.5 \mathrm{~s}^{-1}$ and $C_{a}$ is the $\mathrm{Ca}^{2+}$ concentration at the apical membrane. $V_{a}$ is the membrane potential of the apical membrane. The total current through the $\mathrm{Cl}^{-}$channels is then given by

$I_{\mathrm{Cl}}=g_{\mathrm{cl}} O\left(V_{a}-V_{\mathrm{Cl}}\right)$,

where $g_{\mathrm{Cl}}=31.4 \mathrm{nS}$ is the maximum whole cell conductance found by Arreola et al. (1996). $V_{\mathrm{Cl}}$ is the Nernst potential given by

$V_{\mathrm{Cl}}=\frac{R T}{z_{\mathrm{Cl}} F} \log \left(\frac{[\mathrm{Cl}]_{l}}{[\mathrm{Cl}]_{i}}\right)$,

where $[\mathrm{Cl}]_{l}$ and $[\mathrm{Cl}]_{i}$ are the $\mathrm{Cl}^{-}$concentrations in the lumen and cytosol respectively and $z_{\mathrm{Cl}}=-1$ is the valence of $\mathrm{Cl}^{-}$, $R=8.315 \mathrm{~J} \mathrm{~mol}^{-1} \mathrm{~K}^{-1}, T=310 \mathrm{~K}$ and $F=96,490 \mathrm{C} \mathrm{mol}^{-1}$.

\subsection{The effect of wave speed on fluid secretion rate in a model with} time-dependent $\mathrm{Cl}^{-}$channels

The effect of wave speed on fluid flow is investigated using the same method of varying the time difference between the apical and basal $\mathrm{Ca}^{2+}$ peaks described in Section 4 . When time dependence of the $\mathrm{Cl}^{-}$channel is added to the model we find that a maximum secretion rate occurs with a small positive time difference between the $\mathrm{Ca}^{2+}$ peaking at the apical and basal membranes (see Fig. 4). $\mathrm{Ca}^{2+}$ waves are simulated with a sinusoidal function with mean $150 \mathrm{nM}$, amplitude $100 \mathrm{nM}$ and a period of $7 \mathrm{~s}$.

In Fig. 4 it can be seen that maximum secretion occurs when the $\mathrm{Ca}^{2+}$ wave peaks at the apical membrane $0.2 \mathrm{~s}$ before the basal membrane. This roughly equates to an apical to basal wave 


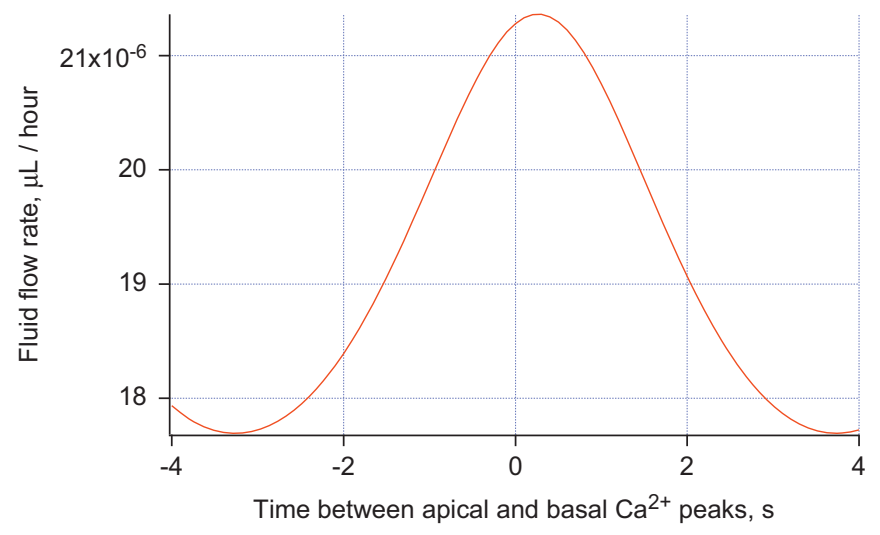

Fig. 4. Fluid flow rate against time difference between apical and basal $\mathrm{Ca}^{2+}$ peaks in a model with time-dependent $\mathrm{Cl}^{-}$channels. Maximum fluid flow occurs when $\mathrm{Ca}^{2+}$ peaks at the apical membrane $0.2 \mathrm{~s}$ before the basal membrane.

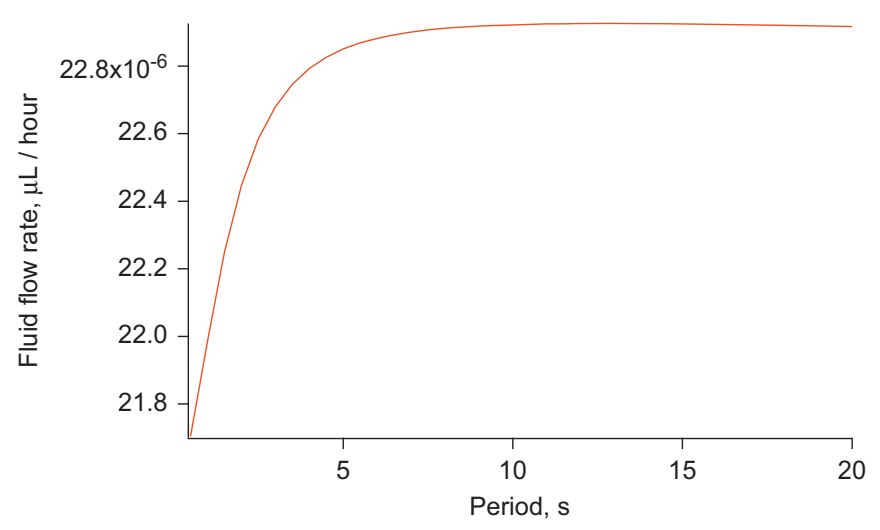

Fig. 5. Fluid flow rate against period of $\mathrm{Ca}^{2+}$ oscillations. The maximum fluid flow rate occurs with long period oscillations.

with a speed of $125 \mu \mathrm{m} / \mathrm{s}$, assuming a cell size of $25 \mu \mathrm{m}$ from apical to basal membrane. This is much faster than the observed wave speed of $27.81 \mu \mathrm{m} / \mathrm{s}$ seen by Won et al. (2007).

\subsection{The effect of wave period on fluid secretion rate in a model with time-dependent $\mathrm{Cl}^{-}$channels}

In Fig. 5 a sinusoidal function is used to simulate $\mathrm{Ca}^{2+}$ at the two membranes with a mean of $100 \mathrm{nM}$ and an amplitude of $50 \mathrm{nM}$.

Fig. 5 shows that as the period of the $\mathrm{Ca}^{2+}$ waves is increased the average saliva secretion rate increases. If $\mathrm{Ca}^{2+}$ oscillates quickly the time-dependent $\mathrm{Cl}^{-}$channel will lag behind the current $\mathrm{Ca}^{2+}$ concentration. This results in less than maximum fluid flow. As the $\mathrm{Ca}^{2+}$ oscillation period is increased we find that fluid flow reaches a maximum. It should be noted that the rate of secretion changes very little despite large changes in oscillation period with the least efficient rate being only $96 \%$ of the most efficient period.

5.3. The effect of mean $\mathrm{Ca}^{2+}$ on fluid secretion rate in a model with time-dependent $\mathrm{Cl}^{-}$channels

The effect of mean $\mathrm{Ca}^{2+}$ on fluid flow is seen in Fig. 6. As the mean $\mathrm{Ca}^{2+}$ concentration increases the amount of secretion increases. The profile of fluid flow as $\mathrm{Ca}^{2+}$ increases takes a sigmoidal shape, initially increasing rapidly at low $\mathrm{Ca}^{2+}$ concentrations and levelling off as the ion channel open probability approaches 1 . A very large difference is observed in secretion

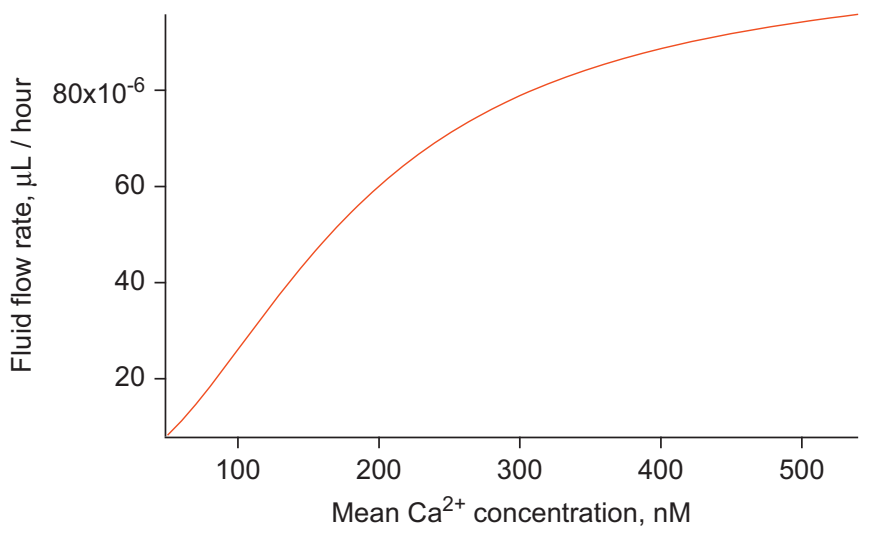

Fig. 6. Fluid flow rate against mean $\mathrm{Ca}^{2+}$ concentration. Larger mean $\mathrm{Ca}^{2+}$ concentrations increase secretion rate. Simulations are completed using a sine function with $20 \mathrm{nM}$ amplitude and $7 \mathrm{~s}$ period.

rates with a high $\mathrm{Ca}^{2+}$ concentration secreting almost 10 times the volume of low concentrations.

\subsection{The effect of oscillation amplitude on fluid secretion rate in a model with time-dependent $\mathrm{Cl}^{-}$channels}

The effect of oscillation amplitude on fluid flow is investigated. With a mean $\mathrm{Ca}^{2+}$ concentration of $100 \mathrm{nM}$, increasing the amplitude of oscillations increases the rate of secretion. This can be seen in Fig. 7(a). If instead the mean $\mathrm{Ca}^{2+}$ is increased to $300 \mathrm{nM}$ the opposite is true, Fig. 7(b), with increasing oscillation amplitude reducing secretion. This result can be explained by the profile of fluid flow with mean $\mathrm{Ca}^{2+}$ seen in Fig. 6. At low $\mathrm{Ca}^{2+}$ concentrations the function is convex. Jensen's inequality states

$E[f(x)] \geq f(E[x])$,

where $E$ is the expectation and $f$ is a convex function. If we consider an oscillating function of $\mathrm{Ca}^{2+}$, this inequality says that the average secretion rate for some oscillating function of $\mathrm{Ca}^{2+}$ is greater than the rate of secretion at the mean $\mathrm{Ca}^{2+}$ concentration. Or equivalently, we expect a larger amplitude to give us greater secretion than a constant $\mathrm{Ca}^{2+}$ concentration.

With a larger mean $\mathrm{Ca}^{2+}$ concentration the profile of secretion with $\mathrm{Ca}^{2+}$ becomes concave and the converse is true with larger amplitude causing a reduction in secretion. At a mean $\mathrm{Ca}^{2+}$ concentration of around $120 \mathrm{nM}$ the secretion rate as a function of mean $\mathrm{Ca}^{2+}$ is neither convex nor concave. Here amplitude has no significant effect on secretion (result not shown).

\section{Discussion}

$\mathrm{Ca}^{2+}$ signals involving oscillations are commonly found in biological systems, and are thought to enable a larger bandwidth of signalling. We have found that each of the investigated properties of $\mathrm{Ca}^{2+}$ waves are capable of altering the rate of saliva secretion to differing degrees.

We find $\mathrm{Ca}^{2+}$ oscillation frequency to be inefficient at regulating secretion rate, with only a $4 \%$ change in secretion over a large range of frequencies. Any difference in secretion is due to the time-dependence of the $\mathrm{Cl}^{-}$channel as no change is observed when this is absent from the model. Gray (1988) finds that given a large range of applied agonist concentrations the parotid acinar cells oscillated with a reasonably constant frequency, potentially supporting the idea that frequency encoding is unimportant in the parotid acinar cell. It is worth noting the shape of Fig. 5 . As the period is increased a plateau is reached. Bruce et al. (2002) report 

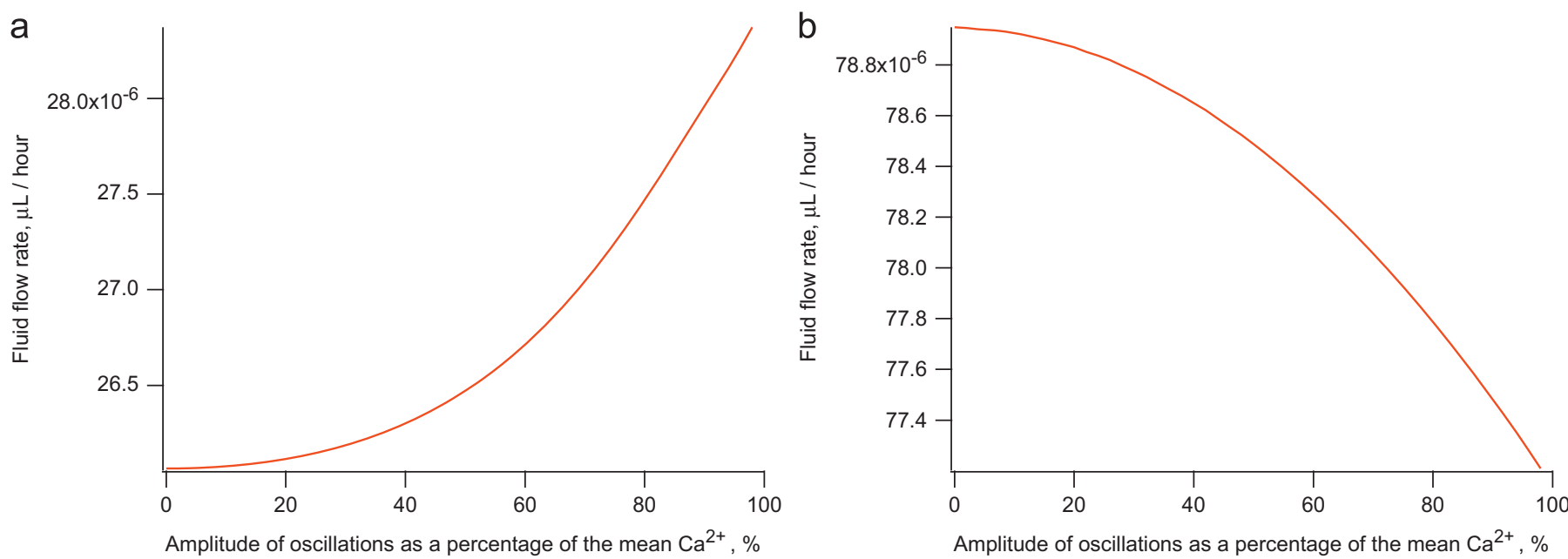

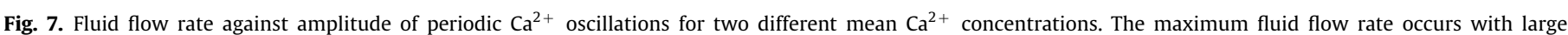

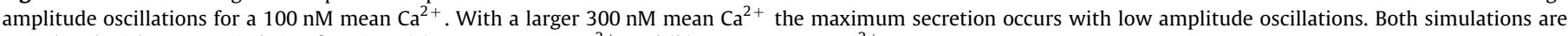
completed with a $7 \mathrm{~s}$ period sine function: (a) $100 \mathrm{nM}$ mean $\mathrm{Ca}^{2+}$ and (b) $300 \mathrm{nM}$ mean $\mathrm{Ca}^{2+}$.

7-11 $\mathrm{Ca}^{2+}$ oscillations per minute in parotid acinar cells giving a period of 5.5-8.5 $\mathrm{s}$. This physiologically realistic range for oscillations lies just at the start of the plateau maximising efficiency of secretion.

As the $\mathrm{Ca}^{2+}$ wave speed is changed a noticeable change in secretion rate occurs with the least efficient wave speed secreting $83 \%$ of the maximum secretion rate that is obtained with the most efficient wave speed. Our model with time-dependent $\mathrm{Cl}^{-}$channels predicts apical to basal $\mathrm{Ca}^{2+}$ waves to be the most efficient, however, the most efficient secretion is predicted for a wave speed much faster than observed experimentally by Won et al. (2007). The model used for this analysis has $\mathrm{Cl}^{-}$channels in the apical membrane and $\mathrm{K}^{+}$in the basal membrane. There is evidence, both experimental and theoretical (Almassy et al., 2012; Palk et al., 2010) that apical $\mathrm{K}^{+}$channels are found in parotid acinar cells. These $\mathrm{K}^{+}$channels are thought to be of the maxi-K type. It is possible that the addition of these $\mathrm{K}^{+}$channels to the model with their time dependence could make slower wave speeds more efficient to parotid acinar cell function. Future work would require a detailed study of the activation of maxi-K channels by $\mathrm{Ca}^{2+}$ in order for this to be properly resolved.

The experimentally observed wave speed of $27.81 \mu \mathrm{m} / \mathrm{s}$ seen by Won et al. (2007) is found to remain roughly constant in parotid acinar cells for varying amounts of stimulation, and therefore it seems unlikely that wave speed is used as a signalling mechanism. This wave speed is very similar to $\mathrm{Ca}^{2+}$ waves observed in other mammalian cell types by Jaffe (1991), with only cardiac myocytes displaying much greater wave speeds. It seems possible that a similar wave generation mechanism in different cell types might limit wave speeds to this narrow range. One might conjecture that $\mathrm{Ca}^{2+}$ waves travel at a speed which maximises fluid secretion or, alternatively, that the ion channels responsible for fluid regulation have adapted to maximise secretion for this constrained wave speed.

The effect of $\mathrm{Ca}^{2+}$ oscillation amplitude on secretion is dependent on the mean $\mathrm{Ca}^{2+}$ concentration, with increasing amplitude increasing secretion at low $\mathrm{Ca}^{2+}$ concentrations and decreasing secretion as the mean $\mathrm{Ca}^{2+}$ increases. Gray (1988) reports a large range of oscillation amplitudes seen experimentally and thus it is unclear what role amplitude might have in signalling.

By far the most significant mechanism for signalling is the mean $\mathrm{Ca}^{2+}$ concentration. Here the flow rate for low $\mathrm{Ca}^{2+}$ concentration is less than $10 \%$ what is seen for the highest concentration. Foskett and Melvin (1989) find a resting level of $\mathrm{Ca}^{2+}$ as $59 \mathrm{nM}$, increasing to $474 \mathrm{nM}$ when stimulated with carbachol. According to our model this would result in a 10 -fold increase in secretion. Experimentally an increase between 6 and 13 fold is seen between resting and stimulated salivary glands (Ben-Aryeh et al., 1986; Heft and Baum, 1984).

By avoiding a detailed spatial model, wave properties are easily isolated and investigated for their effect on secretion. Several assumptions are made in using this simplified approach. Changing a global variable, such as the wave speed, is assumed to affect the apical and basal regions equally and not to affect other variables. If we were to alter the wave speed experimentally, perhaps by inhibiting the $\mathrm{Ca}^{2+}$ release channels, we might expect the profile of the oscillations at the two membranes to change. It is also likely that the frequency, amplitude and mean $\mathrm{Ca}^{2+}$ concentration would also be changed.

In Section 5 we consider the time-dependence of the $\mathrm{Cl}^{-}$ channel gating using the experimental data and model of Arreola et al. (1996). There are other time-dependent processes that have not been included in this analysis. As previously mentioned, further data is needed for the inclusion of timedependent maxi-K channels. Membrane mechanics and fluid dynamics are also likely to add time-dependent effects to the model, but are not considered due to their complexity.

The overall aim of our research is to understand the regulation of saliva secretion across temporal and spatial scales from individual ion channels to whole gland secretion rates. To create a multiscale model of saliva secretion we must decide what detail to include and what to simplify. Given that $\mathrm{Ca}^{2+}$ waves are found experimentally, a spatial modelling approach might be taken using partial differential equations to solve for $\mathrm{Ca}^{2+}$. However, unless we are particularly interested in how $\mathrm{Ca}^{2+}$ waves arise then this detailed spatial model will be numerically costly and produce large amounts of data which are not required. A conclusion from this analysis is that a detailed model of $\mathrm{Ca}^{2+}$ waves is unlikely to result in improved results relating to the rate of fluid secretion. By far the most important signalling mechanism is found to be the mean $\mathrm{Ca}^{2+}$ concentration. Therefore it is our opinion than a compartment model using ordinary differential equations with homogeneous $\mathrm{Ca}^{2+}$ oscillations is sufficient when considering secretion rate as the most important model variable. Going further, if mean secretion rate is the only model concern and extreme computational constraints existed, perhaps considering a whole-organ model, it would even be possible to ignore all 
oscillations completely and just consider $\mathrm{Ca}^{2+}$ as a constant function of agonist stimulation.

On the topic of signal transduction we might hypothesise that the process of salivation does not require the complex signal encoding that is seen in some other cell types. It seems unlikely that we must signal for an exact saliva secretion rate. If accuracy in the flow rate is not required then an increase in mean $\mathrm{Ca}^{2+}$ might be all that is required as a signalling mechanism. We might further hypothesise that the other experimentally observed wave properties, such as oscillation frequency and wave speed, might be tuned to values which offer the maximum efficiency in secretion for a given mean $\mathrm{Ca}^{2+}$ concentration.

\section{Acknowledgements}

This work was supported by National Institutes of Health Grant R01-DE19245.

\section{Appendix A. Model equations}

\section{A.1. Fluid flow model}

A summary of the main differential equations in the fluid flow model is included below. We use the following subscript notation with $[\mathrm{Cl}]_{i}$, $[\mathrm{Cl}]_{l}$ and $[\mathrm{Cl}]_{e}$ denoting the $\mathrm{Cl}^{-}$concentration in the cytosol, lumen and interstitium respectively. For full details and parameter values see Palk et al. (2010), we choose to not include apical $\mathrm{K}^{+}$channels in this analysis and therefore set $\alpha_{K}=1$. Differential equations for the cytosolic concentrations are as follows:

$\frac{d\left([\mathrm{Cl}]_{i} w\right)}{d t}=-\frac{I_{\mathrm{Cl}}}{z_{\mathrm{Cl}} F}+2 J_{\mathrm{NKCC}}$,

$\frac{d\left([\mathrm{Na}]_{i} w\right)}{d t}=-3 J_{\mathrm{NaK}}+J_{\mathrm{NKCC}}$,

$\frac{d\left([\mathrm{~K}]_{i} w\right)}{d t}=2 J_{\mathrm{NaK}}+J_{\mathrm{NKCC}}-\frac{I_{\mathrm{K}}}{z_{\mathrm{K}} F}$,

where

$I_{\text {tight }}=\frac{V_{a}-V_{b}}{R_{\text {tight }}}$

is the current through the tight junction.

Lumenal ionic concentrations are given by the following differential equations:

$w_{L} \frac{d\left([\mathrm{Na}]_{l}\right)}{d t}=\frac{g_{\mathrm{t}, \mathrm{Na}} I_{\text {tight }}}{z_{\mathrm{Na}} F}-q_{\mathrm{tot}}[\mathrm{Na}]_{l}$,

$w_{L} \frac{d\left([\mathrm{~K}]_{l}\right)}{d t}=\frac{\left(1-g_{\mathrm{t}, \mathrm{Na}}\right) I_{\mathrm{tight}}}{z_{\mathrm{K}} F}-q_{\mathrm{tot}}[\mathrm{K}]_{l}$,

$w_{L} \frac{d\left([\mathrm{Cl}]_{l}\right)}{d t}=\frac{I_{\mathrm{Cl}}}{z_{\mathrm{Cl}} F}-q_{\mathrm{tot}}[\mathrm{Cl}]_{l}$.

Equations for the basal and apical membrane potentials are

$C_{m} \frac{d V_{b}}{d t}=-I_{\mathrm{K}}-F J_{\mathrm{NaK}}+I_{\text {tight }}$,

$C_{m} \frac{d V_{a}}{d t}=-I_{\mathrm{Cl}}-I_{\text {tight }}$

The fluid flow across the apical membrane is

$q_{a}=R T L_{\mathrm{Pa}}\left([\mathrm{Cl}]_{l}+[\mathrm{Na}]_{l}+[\mathrm{K}]_{l}-\left([\mathrm{Cl}]_{i}+[\mathrm{Na}]_{i}+[\mathrm{K}]_{i}+[\mathrm{Ca}]_{i}+\frac{x}{w}\right)\right)$, where $[\mathrm{Ca}]_{i}$ is the mean $\mathrm{Ca}^{2+}$ concentration throughout the cytosol given by

$[\mathrm{Ca}]_{i}=\frac{C_{a}+C_{b}}{2}$,

where $C_{a}$ and $C_{b}$ are the apical and basal $\mathrm{Ca}^{2+}$ concentrations respectively.

The basal fluid flow $q_{b}$ and paracellular fluid flow $q_{\text {tight }}$ are given respectively as

$q_{b}=R T L_{\mathrm{Pb}}\left([\mathrm{Cl}]_{i}+[\mathrm{Na}]_{i}+[\mathrm{K}]_{i}+[\mathrm{Ca}]_{i}+\frac{x}{w}-\left([\mathrm{Cl}]_{e}+[\mathrm{Na}]_{e}+[\mathrm{K}]_{e}\right)\right)$,

$q_{\text {tight }}=R T L_{\mathrm{Pt}}\left([\mathrm{Cl}]_{l}+[\mathrm{Na}]_{l}+[\mathrm{K}]_{l}-\left([\mathrm{Cl}]_{e}+[\mathrm{Na}]_{e}+[\mathrm{K}]_{e}\right)\right)$.

The total secretion is then given by the sum of the paracellular and transcellular components:

$q_{\mathrm{tot}}=q_{a}+q_{\mathrm{tight}}$.

The cell volume is governed by the balance of incoming and outgoing fluid flow:

$\frac{d w}{d t}=q_{b}-q_{a}$.

Model paramaters can be seen Table A1. For details of the model derivation see Palk et al. (2010).

\section{A.2. $\mathrm{Cl}^{-}$channels}

For the analysis in Section 4 a steady-state model of $\mathrm{Cl}^{-}$ channel gating from Arreola et al. (1996) is used (for details of the non-steady-state model see Section 5). Here the $\mathrm{Cl}^{-}$channel steady-state open probability is given as

$P_{\mathrm{Cl}}=\frac{1}{1+K_{2}\left(K_{1}^{2} / C_{a}^{2}+K_{1} / C_{a}+1\right)}$,

where $C_{a}$ is the $\mathrm{Ca}^{2+}$ concentration at the apical membrane and $K_{1}=214 \exp \left(\frac{-0.13 F V_{a}}{R T}\right) n M$,

$K_{2}=0.58 \exp \left(\frac{-0.24 F V_{a}}{R T}\right)$

Here $V_{a}$ is the membrane potential of the apical membrane. Total current through the $\mathrm{Cl}^{-}$channels is then given by

$I_{\mathrm{Cl}}=g_{\mathrm{cl}} P_{\mathrm{Cl}}\left(V_{a}-V_{\mathrm{Cl}}\right)$.

$g_{\mathrm{Cl}}$ is the maximum whole cell conductance, $31.4 \mathrm{nS}$, found by Arreola et al. (1996). $V_{\mathrm{Cl}}$ is the Nernst potential given by

$V_{\mathrm{Cl}}=\frac{R T}{Z_{\mathrm{Cl}} F} \log \left(\frac{\left[\mathrm{Cl}_{l}\right.}{\left[\mathrm{Cl}_{i}\right.}\right)$.

$z_{\mathrm{Cl}}=-1$ is the valence of $\mathrm{Cl}^{-}, R=8.315 \mathrm{~J} \mathrm{~mol}^{-1} \mathrm{~K}^{-1}, T=310 \mathrm{~K}$ and $F=96,490 \mathrm{C} \mathrm{mol}^{-1}$.

\section{A.3. $\mathrm{K}^{+}$channels}

We use the model of Takahata et al. (2003). The steady-state open probability of the $\mathrm{K}^{+}$channel at the basal membrane is given as

$P_{K}=\frac{1}{1+\left(K_{d} / C_{b}\right)^{n H}}$,

where $C_{b}$ is the $\mathrm{Ca}^{2+}$ concentration at the basal membrane and $n H=2.54$ and $K_{d}=0.182 \mu \mathrm{M}$. $K_{d}$ is modified from the value found by Takahata et al. (2003) of $K_{d}=0.43 \mu \mathrm{M}$ to give a small open probability at steady state $\mathrm{Ca}^{2+}$ concentrations. 


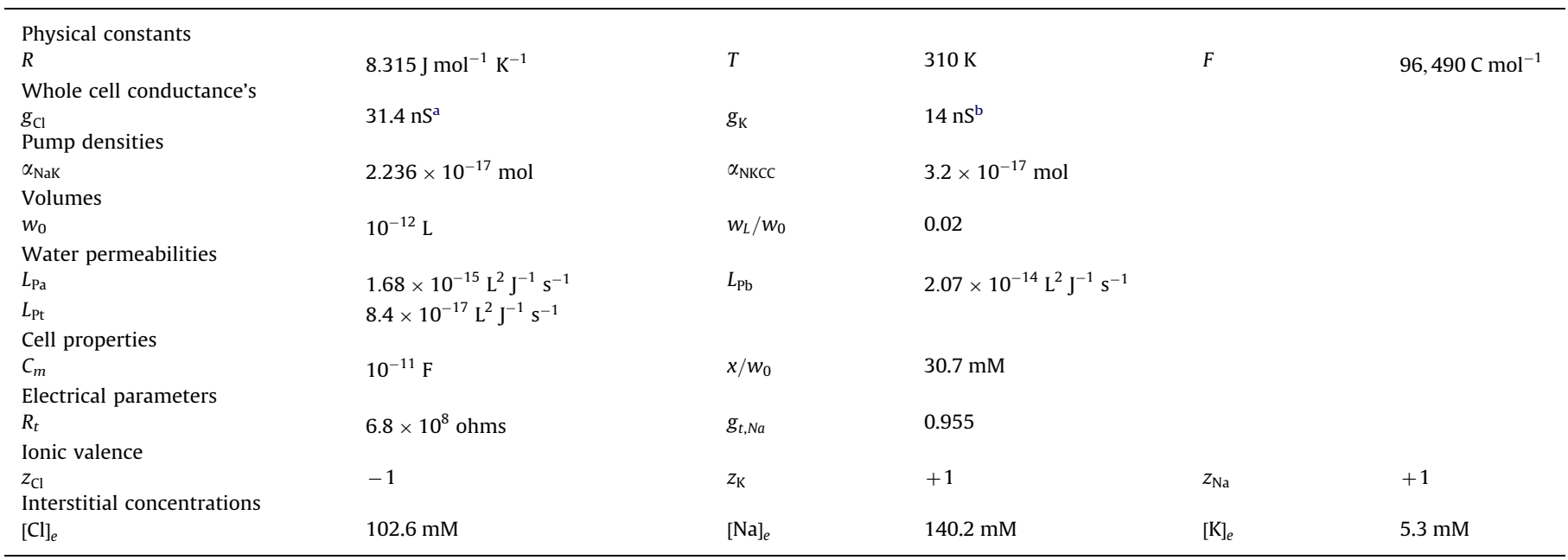

Other parameters are physical constants or model fits chosen to give the correct steady-state concentrations and membrane potentials.

a From Arreola et al. (1996).

b From Thompson and Begenisich (2006).

The current through the $\mathrm{K}^{+}$channel at the basolateral membrane, $I_{K}$, is given by

$I_{\mathrm{K}}=g_{K} P_{K}\left(V_{b}-V_{\mathrm{K}}\right)$,

where $g_{K}$ is the maximum whole cell conductance of $14 \mathrm{nS}$, the value found by Thompson and Begenisich (2006). $V_{K}$ is the nernst potentials of the basolateral membrane given by

$V_{\mathrm{K}}=\frac{R T}{z_{K} F} \log \left(\frac{[\mathrm{K}]_{e}}{[\mathrm{~K}]_{i}}\right)$.

Here $z_{K}=+1$ is the valence of $K^{+}$.

\section{A.4. $\mathrm{Na}^{+}-\mathrm{K}^{+}-$ATPase simplification}

As in Palk et al. (2010) a simplified model of the $\mathrm{Na}^{+}-\mathrm{K}^{+}-$ATPase is used with the steady-state flux as follows:

$v_{\mathrm{NaK}}=r \frac{[K]_{e}^{2}[\mathrm{Na}]_{i}^{3}}{[K]_{e}^{2}+\alpha[\mathrm{Na}]_{i}^{3}}$.

With $r=1.305 \times 10^{6} \mathrm{~s}^{-1} \mathrm{mM}^{-3}$ and $\alpha=0.647 \mathrm{mM}^{-1}$. $J_{\mathrm{NaK}}=$ $\alpha_{\mathrm{NaK}} v_{\mathrm{NaK}}$, where $\alpha_{\mathrm{NaK}}=2.236 \times 10^{-17} \mathrm{~mol}$ is the density of the $\mathrm{Na}^{+}-\mathrm{K}^{+}-$ATPase exchanger.

\section{A.5. $\mathrm{Na}^{+}-\mathrm{K}^{+}-2 \mathrm{Cl}^{-}$cotransporter simplification}

As in Palk et al. (2010) a simplified model of the $\mathrm{Na}^{+}-\mathrm{K}^{+}-$ $2 \mathrm{Cl}^{-}$cotransporter is used with the steady-state flux as follows:

$v_{\mathrm{NKCC}}=r_{\mathrm{NKCC}} \frac{1-\alpha_{1}[\mathrm{Na}]_{i}[K]_{i}[\mathrm{Cl}]_{i}^{2}}{K_{\mathrm{NKCC}}+\alpha_{2}[\mathrm{Na}]_{i}[K]_{i}[\mathrm{Cl}]_{i}^{2}}$,

where $r_{\mathrm{NKCC}}=4.31 \mathrm{~s}^{-1}, \alpha_{1}=1.2755 \times 10^{5}, \alpha_{2}=3.7894 \times 10^{4}$ and $K_{\mathrm{NKCC}}=0.0282 \mathrm{mM}^{4}$. $J_{\mathrm{NKCC}}=v_{\mathrm{NKCC}} \alpha_{\mathrm{NKCC}} \quad$ where $\alpha_{\mathrm{NKCC}}=3.2 \times$ $10^{-17} \mathrm{~mol}$ is the membrane density of the cotransporter.

\section{Appendix B. Approximate analysis of model equations: synchronous $\mathrm{Ca}^{2+}$ waves produce a local maximum for fluid secretion}

Here we seek to show that the fluid secretion model with steady-state ion channels seen in Section 2 has a maximum secretion rate when $\mathrm{Ca}^{2+}$ oscillations are synchronous at apical and basal membranes. In order to do this we must make some assumptions. First we make the assumption that the membrane potentials are at quasi-steady-state, which we can justify given the very small membrane capacitance $C_{m}$. This gives

$C_{m} \frac{d V_{a}}{d t}=-I_{\mathrm{Cl}}-I_{\text {tight }}=0$

and

$C_{m} \frac{d V_{b}}{d t}=-I_{K}-F J_{\mathrm{Nak}}+I_{\text {tight }}=0$.

Now we substitute the definitions for the currents, $I_{K}, I_{\mathrm{Cl}}$ and $I_{\text {tight }}$ into Eqs. (B.1) and (B.2), giving

$-g_{\mathrm{cl}} P_{\mathrm{cl}}\left(V_{a}-V_{\mathrm{Cl}}\right)=\left(V_{a}-V_{b}\right) / R_{\text {tight }}$

and

$g_{k} P_{k}\left(V_{b}-V_{k}\right)+F J_{\mathrm{Nak}}=\left(V_{a}-V_{b}\right) / R_{\mathrm{tight}}$

If we solve both these equations simultaneously we can get expressions for the membrane potentials $V_{a}$ and $V_{b}$. During simulations it is found that, for near isosmotic fluid secretion, fluid flow is proportional to the current through the tight junction, see Maclaren et al. (2012). The tight junctional current is given by $\left(V_{a}-V_{b}\right) / R_{\text {tight }}$, we use this as follows:

flow $\propto \frac{V_{a}-V_{b}}{R_{\text {tight }}}=\frac{P_{\mathrm{CL}} P_{K}\left(V_{\mathrm{Cl}}-V_{k}\right)+P_{\mathrm{CL}} F J_{\mathrm{Nak}}}{P_{K}+P_{K} R_{\mathrm{tight}} P_{\mathrm{CL}}+P_{\mathrm{CL}}}$.

Here we have used the notations $P_{\mathrm{CL}}=P_{\mathrm{cl}} g_{\mathrm{Cl}}$ and $P_{K}=P_{k} g_{k}$.

\section{B.1. Periodic functions}

We will make the assumption that during the course of one $\mathrm{Ca}^{2+}$ wave both $P_{C L}$ and $P_{K}$ are periodic functions. We take this assumption further to make these both the same periodic function with a phase difference $\delta$. We also assume $V_{\mathrm{Cl}}$ and $V_{k}$ stay approximately constant, a valid assumption if changes in ionic concentrations are small. We then denote

$f(t)=\frac{1}{P_{K}\left(V_{\mathrm{Cl}}-V_{k}\right)}, \quad f(t+\delta)=\frac{1}{P_{\mathrm{CL}}\left(V_{\mathrm{Cl}}-V_{k}\right)}$,

$\gamma=F J_{\mathrm{Nak}} \quad$ and $\quad A=\frac{R_{\mathrm{tight}}}{\left(V_{\mathrm{Cl}}-V_{k}\right)}$. 
The expression for fluid flow then becomes

$f l o w \propto \frac{1+\gamma f(t)}{f(t)+f(t+\delta)+A}$,

where $A$ and $\gamma$ are positive constants and $f(t)$ is any periodic function, period $T$. Now we would like to see how the average flow over the course of one period, $T$, depends on the phase difference $\delta$.

We define $I$ to be the total flow over a period, $T$,

$I=\int_{0}^{T} \frac{1+\gamma f(t)}{A+f(t)+f(t+\delta)} d t$

We would like to find when this expression has a maximum and minimum. Taking the derivative with respect to $\delta$

$\frac{\partial I}{\partial \delta}=-\int_{0}^{T} \frac{(1+\gamma f(t)) f^{\prime}(t+\delta)}{(A+f(t)+f(t+\delta))^{2}} d t$.

We predict a maximum at $\delta=0$, so looking at the partial derivative here,

$$
\begin{aligned}
\left.\frac{\partial I}{\partial \delta}\right|_{\delta=0}=-\int_{0}^{T} \frac{(1+\gamma f(t)) f^{\prime}(t)}{(A+2 f(t))^{2}} d t \\
\quad=\frac{1}{2(A+2 f(t))}-\frac{\gamma}{4} \ln (2 f(t)+A)-\left.\frac{\gamma A}{4(2 f(t)+A)}\right|_{0} ^{T} .
\end{aligned}
$$

As, $2 f(0)=2 f(T)$, being a periodic function, this gives

$\left.\frac{\partial I}{\partial \delta}\right|_{\delta=0}=0$

and therefore a maximum or minimum occurs when there is no phase difference. To determine whether this is a maximum or minimum we look at the second derivative.

$\left.\frac{\partial^{2} I}{\partial \delta^{2}}\right|_{\delta=0}=-\int_{0}^{T} \frac{(1+\gamma f(t)) f^{\prime \prime}(t)}{(A+2 f(t))^{2}} d t+2 \int_{0}^{T} \frac{(1+\gamma f(t)))\left(f^{\prime}(t)\right)^{2}}{(A+f(t))^{3}} d t$ $\equiv I_{1}+I_{2}+I_{3}+I_{4}$,

where

$I_{1}=\int_{0}^{T} \frac{-f^{\prime \prime}(t)}{(A+2 f(t))^{2}} d t$

$I_{2}=2 \int_{0}^{T} \frac{\left(f^{\prime}(t)\right)^{2}}{(A+f(t))^{3}} d t$

$I_{3}=\int_{0}^{T}-\frac{\gamma f(t) f^{\prime \prime}(t)}{(A+2 f(t))^{2}}$

and

$I_{4}=\int_{0}^{T} \frac{2 \gamma f(t)\left(f^{\prime}(t)\right)^{2}}{(A+2 f(t))^{3}}$.

Evaluating the first integral, $I_{1}$, using integration by parts

$I_{1}=-\left.\frac{f^{\prime}(t)}{(A+2 f(t))^{2}}\right|_{0} ^{T}-\int_{0}^{T} \frac{4\left(f^{\prime}(t)\right)^{2}}{(A+2 f(t))^{3}} d t=0-4 \int_{0}^{T} \frac{\left(f^{\prime}(t)\right)^{2}}{(A+2 f(t))^{3}} d t$.

Evaluating the integral $I_{3}$ using integration by parts

$$
\begin{aligned}
I_{3}= & \int_{0}^{T}-\frac{\gamma f(t) f^{\prime \prime}(t)}{(A+2 f(t))^{2}} \\
= & -\left.\frac{\gamma f(t) f^{\prime}(t)}{(A+2 f(t))^{2}}\right|_{0} ^{T}+\int_{0}^{T} \frac{\gamma\left(f^{\prime}(t)\right)^{2}}{(A+2 f(t))^{2}} d t \\
& -\int_{0}^{T} \frac{4 \gamma f(t)\left(f^{\prime}(t)\right)^{2}}{(A+2 f(t))^{3}} d t \\
= & 0+\int_{0}^{T} \frac{\gamma\left(f^{\prime}(t)\right)^{2}}{(A+2 f(t))^{2}} d t
\end{aligned}
$$

$$
-\int_{0}^{T} \frac{4 \gamma f(t)\left(f^{\prime}(t)\right)^{2}}{(A+2 f(t))^{3}} d t
$$

Now

$$
\begin{aligned}
\left.\frac{\partial^{2} I}{\partial \delta^{2}}\right|_{\delta=0}= & -\int_{0}^{T} \frac{2\left(f^{\prime}(t)\right)^{2}}{(A+2 f(t))^{3}} d t-\int_{0}^{T} \frac{2 \gamma f(t)\left(f^{\prime}(t)\right)^{2}}{(A+2 f(t))^{3}} d t \\
& +\int_{0}^{T} \frac{\gamma\left(f^{\prime}(t)\right)^{2}}{(A+2 f(t))^{2}} d t \\
= & -(2-\gamma A) \int_{0}^{T} \frac{\left(f^{\prime}(t)\right)^{2}}{(A+2 f(t))^{3}} d t .
\end{aligned}
$$

If we assume $A$ is a positive constant and that $f(t)$ is a positive function, both valid assumptions, then here we are taking the integral of an expression that is strictly positive. The sign of the second derivative is therefore determined by the expression $(2-\gamma A)$. Looking back to the original notation

$\gamma A=F J_{\mathrm{Nak}} \frac{R_{\mathrm{tight}}}{\left(V_{\mathrm{Cl}}-V_{k}\right)}$.

Under physiological condition the various terms are of the following magnitude:

$\left(V_{\mathrm{Cl}}-V_{\mathrm{K}}\right) \sim O\left(10^{-2}\right)$,

$R_{\text {tight }} \sim O\left(10^{8}\right)$

$F J_{\text {Nak }} \sim O\left(10^{-12}\right)$.

Therefore $\gamma A \sim O\left(10^{-2}\right)$ and

$\left.\frac{\partial^{2} I}{\partial \delta^{2}}\right|_{\delta=0}=-(2-\gamma A) \int_{0}^{T} \frac{\left(f^{\prime}(t)\right)^{2}}{(A+2 f(t))^{3}} d t<0$.

By proving that the second derivative is negative we have shown that $\delta=0$ is a local maximum and thus there is a local maximum in secretion when $\mathrm{Ca}^{2+}$ waves are synchronous at the apical and basal membranes.

\section{References}

Almassy, J., Won, J., Begenisich, T., Yule, D., 2012. Apical $\mathrm{Ca}^{2+}$-activated potassium channels in mouse parotid acinar cells. J. Gen. Physiol. 139 (2), 121-133.

Arreola, J., Melvin, J., Begenisich, T., 1996. Activation of calcium-dependent chloride channels in rat parotid acinar cells. J. Gen. Physiol. 108 (July (1)), 35-47.

Ben-Aryeh, H., Shalev, A., Szargel, R., Laor, A., Laufer, D., Gutman, D., 1986. The salivary flow rate and composition of whole and parotid resting and stimulated saliva in young and old healthy subjects. Biochem. Med. Metabol. Biol. 36 (2), 260-265.

Berridge, M., 1997. The AM and FM of calcium signalling. Nature 386, 759-760.

Bruce, J.I.E., Shuttleworth, T.J., Giovannucci, D.R., Yule, D.I., 2002. Phosphorylation of inositol 145trisphosphate receptors in parotid acinar cells a mechanism for the synergistic effects of cAMP on Ca2 + signaling. J. Biol. Chem. 277 (2), 1340-1348.

De Koninck, P., Schulman, H., 1998. Sensitivity of CaM kinase II to the frequency of $\mathrm{Ca}^{2+}$ oscillations. Science 279 (5348), 227.

Foskett, J., Melvin, J., 1989. Activation of salivary secretion: coupling of cell volume and $\left[\mathrm{Ca}^{2+}\right] i$ in single cells. Science 244 (June (4912)), 1582-1585.

Foskett, J.K., 1990. $\left[\mathrm{Ca}^{2+}\right] i$ modulation of $\mathrm{Cl}$-content controls cell volume in single salivary acinar cells during fluid secretion. AJP_Cell Physiol. 259 (December (6)), C998-C1004.

Gin, E., Crampin, E.J., Brown, D.A., Shuttleworth, T.J., Yule, D.I., Sneyd, J., 2007. A mathematical model of fluid secretion from a parotid acinar cell. J. Theor. Biol. 248 (1), 64-80.

Goldbeter, A., Dupont, G., Berridge, M., 1990. Minimal model for signal-induced $\mathrm{Ca}^{2+}$ oscillations and for their frequency encoding through protein phosphorylation. Proc. Natl. Acad. Sci. USA 87 (4), 1461.

Gray, P., 1988. Oscillations of free cytosolic calcium evoked by cholinergic and catecholaminergic agonists in rat parotid acinar cells. J. Physiol. 406 (1), 35.

Heft, M., Baum, B., 1984. Basic biological sciences unstimulated and stimulated parotid salivary flow rate in individuals of different ages. J. Dent. Res. 63 (10), 1182.

Jaffe, L., 1991. The path of calcium in cytosolic calcium oscillations-a unifying hypothesis. Proc. Natl. Acad. Sci. USA 88 (November (21)), 9883-9887.

Larsen, A., Olsen, L., Kummer, U., 2004. On the encoding and decoding of calcium signals in hepatocytes. Biophys. Chem. 107 (1), 83-99.

Oliver Maclaren, James Sneyd, Edmund Crampin, 2012. Efficiency of Primary Saliva Secretion: An Analysis of Parameter Dependence in Dynamic Single-Cell and 
Acinus Models, with Application to Aquaporin Knockout Studies. Journal of Membrane Biology. 245, Number 1, 29-50, http://dx.doi.org/10.1007/s00232011-9413-3.

Palk, L., Sneyd, J., Shuttleworth, T., Yule, D., Crampin, E., 2010. A dynamic model of saliva secretion. J. Theor. Biol. 266 (4), 625-640, http://dx.doi.org/10.1016/ j.jtbi.2010.06.027.

Sneyd, J., Tsaneva-Atanasova, K., Bruce, J.I.E., Straub, S.V., Giovannucci, D.R., Yule, D.I., 2003. A model of calcium waves in pancreatic and parotid acinar cells. Biophys. J. 85 (3), 1392-1405.

Takahata, T., Hayashi, M., Ishikawa, T., 2003. SK4/IK1-like channels mediate TEAinsensitive, $\mathrm{Ca}^{2+}$-activated $\mathrm{K}+$ currents in bovine parotid acinar cells. AJP_Cell Physiol. 284 (January (1)), C127-C144.
Tang, Y., Othmer, H., 1995. Frequency encoding in excitable systems with applications to calcium oscillations. Proc. Natl. Acad. Sci. 92 (17), 7869.

Thompson, J., Begenisich, T., 2006. Membrane-delimited inhibition of maxi-K channel activity by the intermediate conductance $\mathrm{Ca}^{2}+$-activated $\mathrm{K}$ channel. J. Gen. Physiol. 127 (February (2)), 159-169.

Won, J., Cottrell, W., Foster, T., Yule, D., 2007. $\mathrm{Ca}^{2+}$ release dynamics in parotid and pancreatic exocrine acinar cells evoked by spatially limited flash photolysis. Am. J. Physiol.-Gastrointest. Liver Physiol. 293 (6), G1166.

Zimmermann, B., Walz, B., 1999. The mechanism mediating regenerative intercellular $\mathrm{Ca}^{2+}$ waves in the blowfly salivary gland. EMBO J. 18 (12), 3222-3231. 